\title{
Corrigendum to "Effect of Taichi Softball on Function-Related Outcomes in Older Adults: A Randomized Control Trial"
}

\author{
Lin Luo, ${ }^{1}$ Liye Zou, ${ }^{2,3}$ Qun Fang, ${ }^{2}$ Huiru Wang, ${ }^{4}$ Yang Liu, ${ }^{5}$ \\ Zuguo Tian, ${ }^{6}$ and Yunpeng $\mathrm{Han}^{7}$ \\ ${ }^{1}$ Department of Physical Education, North China Electric Power University, Beijing, China \\ ${ }^{2}$ Department of Physical Education and Health Education, Springfield College, Springfield, MA, USA \\ ${ }^{3}$ Department of Sports Science, Jishou University, China \\ ${ }^{4}$ Department of Physical Education, Shanghai Jiao Tong University, Shanghai, China \\ ${ }^{5}$ Sensorimotor Neurophysiology Laboratory, Indiana University, Bloomington, IN, USA \\ ${ }^{6}$ College of Physical Education, Hunan University, Hunan, China \\ ${ }^{7}$ Department of Physical Education, Guiyang Teacher Training College, Guizhou, China \\ Correspondence should be addressed to Liye Zou; liyezou123@gmail.com
}

Received 9 August 2017; Accepted 12 September 2017; Published 2 November 2017

Copyright (C) 2017 Lin Luo et al. This is an open access article distributed under the Creative Commons Attribution License, which permits unrestricted use, distribution, and reproduction in any medium, provided the original work is properly cited.

In the article titled "Effect of Taichi Softball on FunctionRelated Outcomes in Older Adults: A Randomized Control Trial" [1], the name of the first author was given incorrectly as Lin Lou. The author's name should have been written as Lin Luo. The revised authors' list is shown above.

\section{References}

[1] L. Lou, L. Zou, Q. Fang et al., "Effect of taichi softball on function-related outcomes in older adults: a randomized control trial," Evidence-Based Complementary and Alternative Medicine, vol. 2017, Article ID 4585424, 9 pages, 2017. 


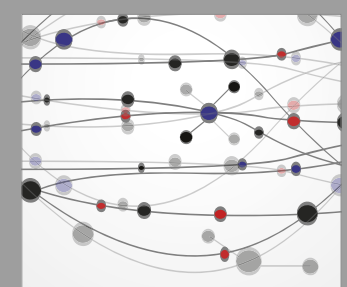

The Scientific World Journal
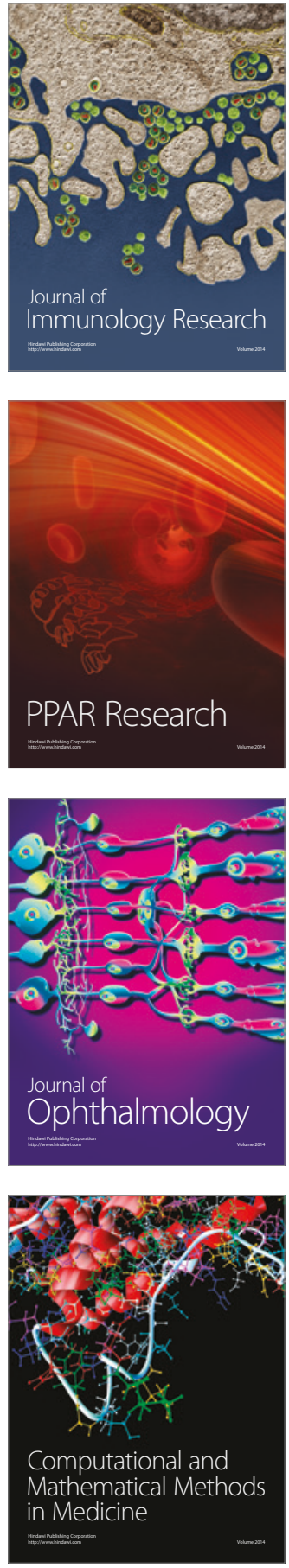

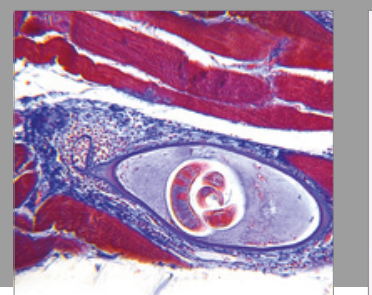

Gastroenterology Research and Practice
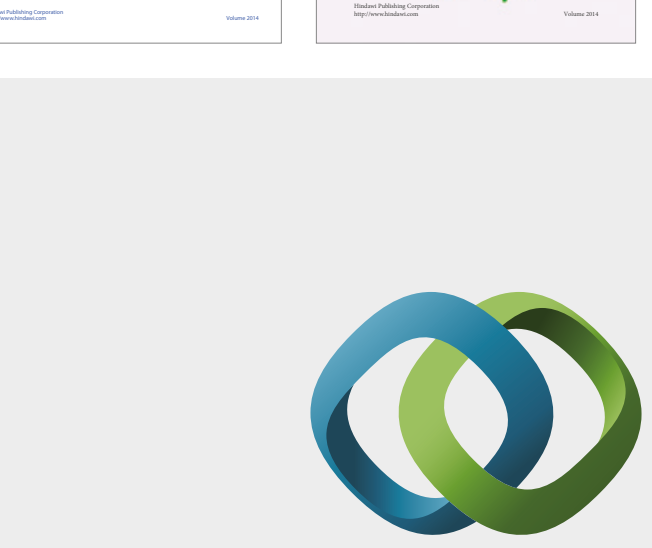

\section{Hindawi}

Submit your manuscripts at

https://www.hindawi.com
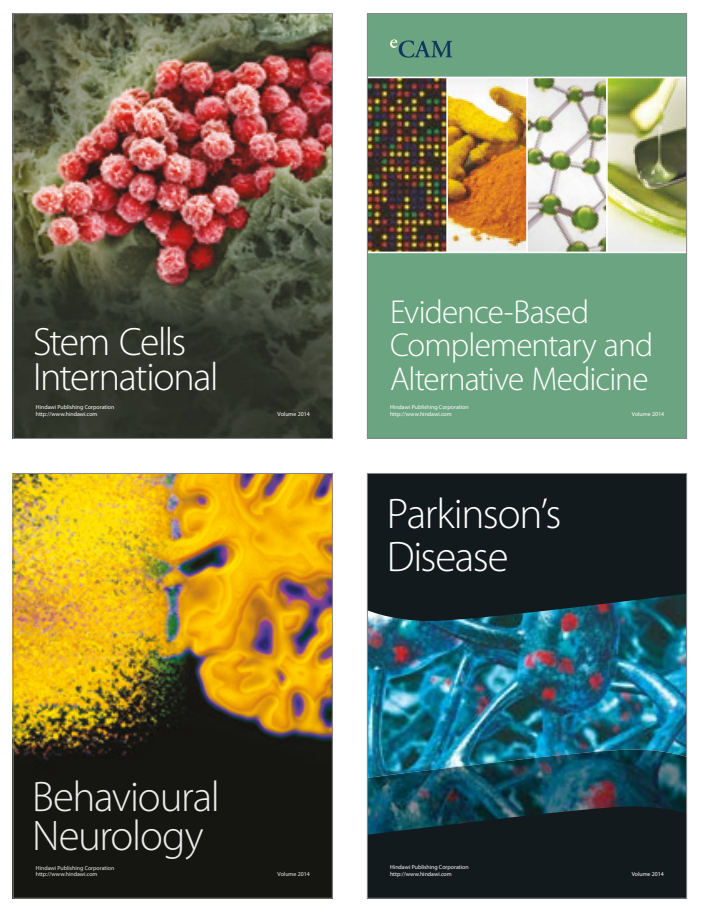
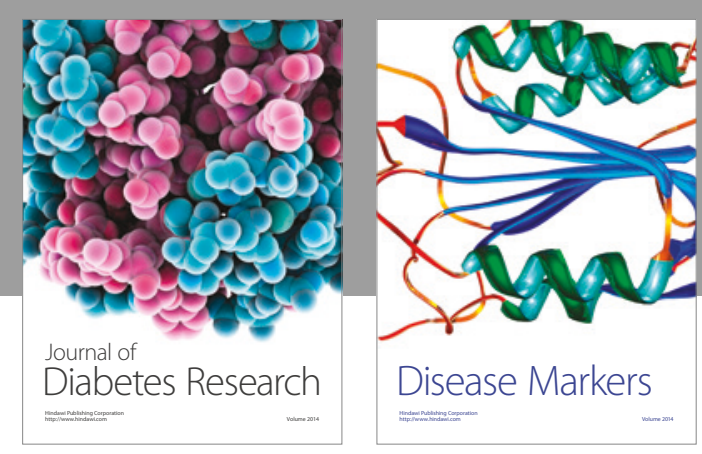

Disease Markers
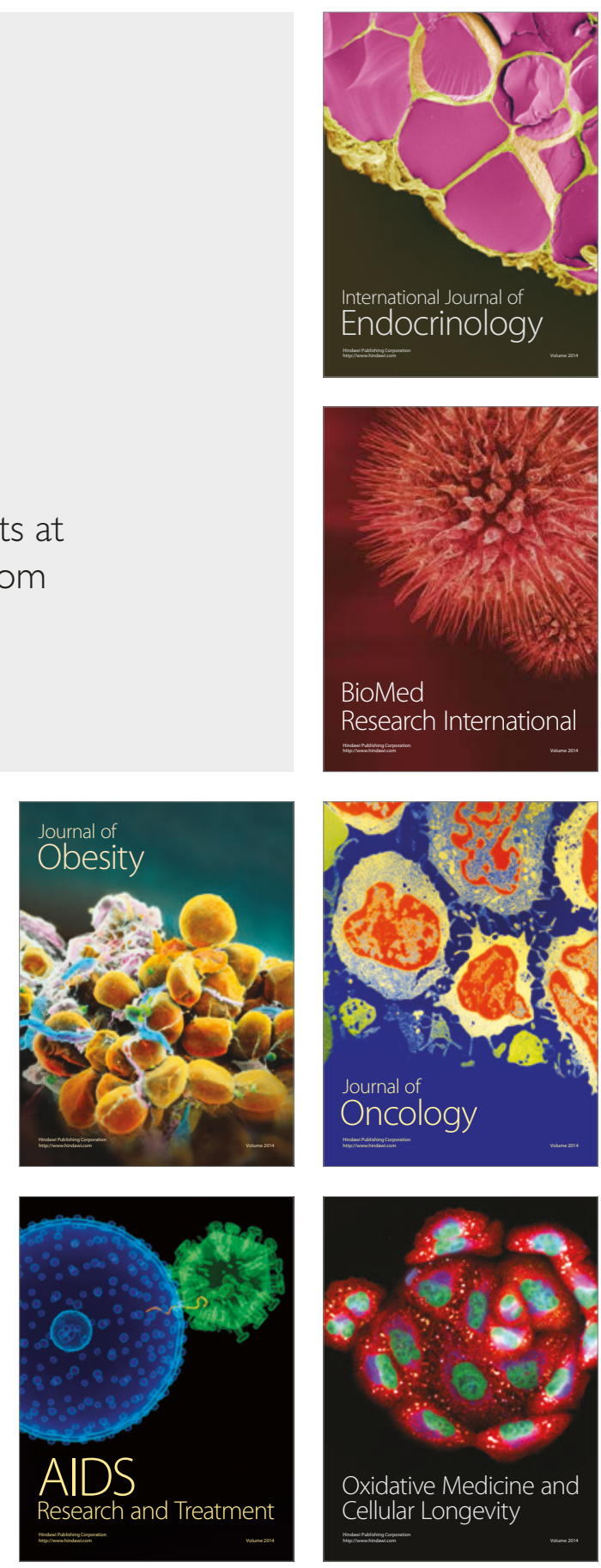